\title{
Neoplasia Papilar Intracolecística. Reporte de un Caso y Revisión de la Evidencia Existente
}

\author{
Intracholecystic Papillary Neoplasia. Report of a Case and Review of Existing Evidence
}

Carlos Manterola ${ }^{1,2}$; Enrique Bellolio ${ }^{3}$;amara Otzen $^{2,4}$ \& Galo Duque ${ }^{5}$

\begin{abstract}
MANTEROLA, C.; BELlOLIO, E.; OTZEN, T. \& DUQUE, G. Neoplasia papilar intracolecística. Reporte de un caso y revisión de la evidencia existente. Int. J. Morphol., 36(4):1485-1489, 2018.

RESUMEN: La neoplasia papilar intracolecística (NPIC), es un tumor compuesto por células neoplásicas preinvasivas, que forman masas de hasta $1,0 \mathrm{~cm}$, clínicamente detectables. El objetivo de este estudio fue reportar un caso de NPIC y revisar la evidencia existente. Se trata de un paciente sexo masculino, de 33 años de edad, asintomático, que en el curso de un examen de salud, se realiza una ecotomografía abdominal, en la que se verifica una lesión polipoide vesicular de 32 x $19 \mathrm{~mm}$ de diámetro. Se programa para colecistectomía electiva, la que se realizó por vía laparoscópica, cirugía que se pudo realizar sin inconvenientes. Una vez extirpado el espécimen, se fue a estudio histopatológico en el que tras un mapeo vesicular se concluye NPIC, colecistitis crónica inespecífica, colesterolosis y pólipos colesterínicos. El paciente ha evolucionado sin inconvenientes. Presentamos un caso de NPIC en un paciente joven, cuyo diagnóstico fue confirmado por anatomía patológica tras una colecistectomía electiva, descartándose la presencia de carcinoma invasivo y displasia de alto grado, por lo que el pronóstico es favorable.
\end{abstract}

PALABRAS CLAVE: Neoplasia papilar-tubular intravesicular; Pólipos neoplásicos; adenoma; Neoplasia papilar; Cáncer de la vesicular biliar; Carcinoma in situ.

\section{INTRODUCCIÓN}

Los tumores compuestos por células neoplásicas preinvasivas y que forman masas clínicamente detectables ( $\geq$ $1,0 \mathrm{~cm}$ ) en el área hepato-bilio-pancreática, han sido denominados "neoplasias papilares intraductales" (BilIN) cuando se desarrollan en los conductos biliares (Albores-Saavedra et al., 2005); y como "neoplasias mucinosas papilares intraductales" o "neoplasias tubulopapilares intraductales" (TPMI), cuando afectan al páncreas (Adsay et al., 2012). Y, en la vesícula biliar, se han descrito lesiones similares, que se han sido denominado de diversas formas (Yamamoto et al., 1988; Nakajo et al., 1990; Albores-Saavedra et al., 1993; Albores-Saavedra et al., 2005; Roa et al., 2006; Escalona et al., 2006; Nagata et al., 2007; Lee et al., 2010; Bhatt et al., 2016), pero que en el último tiempo, responden a la denominación unificada de "neoplasia papilar intracolecística” (NPIC).

Es una neoplasia muy poco frecuente; sin embargo, se ha logrado establecer que se trata de neoplasias preinvasivas, con una clara secuencia "adenoma-carcinoma" (Kozuka et al., 1982; Aldridge \& Bismuth, 1990; Milchgrub et al., 1992; Sessa et al., 1994; Lee et al.; Adsay et al.; Kai, 2016); con un comportamiento biológico diferente al cáncer invasivo de estas localizaciones (Abraham et al., 2003; Adsay et al.); caracterizado por un crecimiento intraluminal asociado a hipersecreción de mucina, al igual que los demás subtipos histológicos: pancreatobiliar, intestinal, gástrico y oncocítico (Bennett et al., 2015).

Existen escasos reportes en la literatura, la mayor parte de los cuales son de casos aislados (Almagro, 1985; Arunabh et al., 1988; Bazira et al., 1998; Kushima et al., 1996; Nagahama et al., 1997; Kosemehmetoglu et al., 2011; Resende et al., 2012; Hashimoto et al., 2014; Michalinos et al., 2016; Páez Cumpa et al., 2017); de reportes de menos de 10 casos (Araki et al., 1988; Diaz-Plasencia et al., 1994; Bennett et al.); y de series entre 16 y hasta 123 casos (Albores-Saavedra et al., 2005; Adsay et al.; Argon et al., 2016), siendo esta última la mayor de todas (Adsay et al.). No obstante ello, entre todas, no suman más de 250 en total, y es posible, que en algunos casos pueda existir duplicidad de información (Tabla I).

\footnotetext{
${ }^{1}$ Departamento de Cirugía, Universidad de La Frontera, Temuco, Chile.

${ }^{2}$ Centro de Excelencia en estudios Morfológicos y Quirúrgicos (CEMyQ), Universidad de La Frontera, Temuco, Chile.

${ }^{3}$ Instituto de Patología Celular y Molecular (IPAT), Temuco, Chile.

${ }^{4}$ Facultad de Ciencias de la Salud, Universidad de Tarapacá, Chile.

${ }^{5}$ Facultad de Medicina, Universidad del Azuay, Cuenca, Ecuador.
} 
El objetivo de este estudio fue reportar un caso de NPIC y revisar la evidencia existente.

Tabla I. Casuística publicada a lo largo del tiempo.

\begin{tabular}{|c|c|c|}
\hline Autor & Año & $\mathrm{N}^{0} \operatorname{casos}$ \\
\hline Almagro & 1985 & 1 \\
\hline Araki et al. & 1988 & 7 \\
\hline Arunabh et al. & 1988 & 1 \\
\hline Bazira et al. & 1998 & 1 \\
\hline Diaz-Plasencia et al. & 1994 & 2 \\
\hline Kushima et al. & 1996 & 1 \\
\hline Nagahama et al. & 1997 & 1 \\
\hline Albores-Saavedra et & 2005 & 16 \\
\hline Kosemehmetoglu et & 2011 & 1 \\
\hline Adsay et al. & 2012 & 123 \\
\hline Resende et al. & 2012 & 1 \\
\hline Hashimoto et al. & 2014 & 1 \\
\hline Bennett et al. & 2015 & 7 \\
\hline Argon et al. & 2016 & 45 \\
\hline Michalinos et al. & 2016 & 1 \\
\hline Páez Cumpa et al. & 2017 & 1 \\
\hline TOTA L & & 210 \\
\hline
\end{tabular}

\section{CASO CLÍNICO}

Presentamos el caso de un paciente de sexo masculino, de 33 años de edad, asintomático, sin antecedentes mórbidos médicos ni quirúrgicos, ni de hábitos tóxicos; que en el curso de un examen rutinario de salud, se le realizó una ecotomografía abdominal, en la que se verificó la existencia de una lesión polipoide de 32 x 19 mm de diámetro, al interior de la vesícula biliar (Fig. 1).

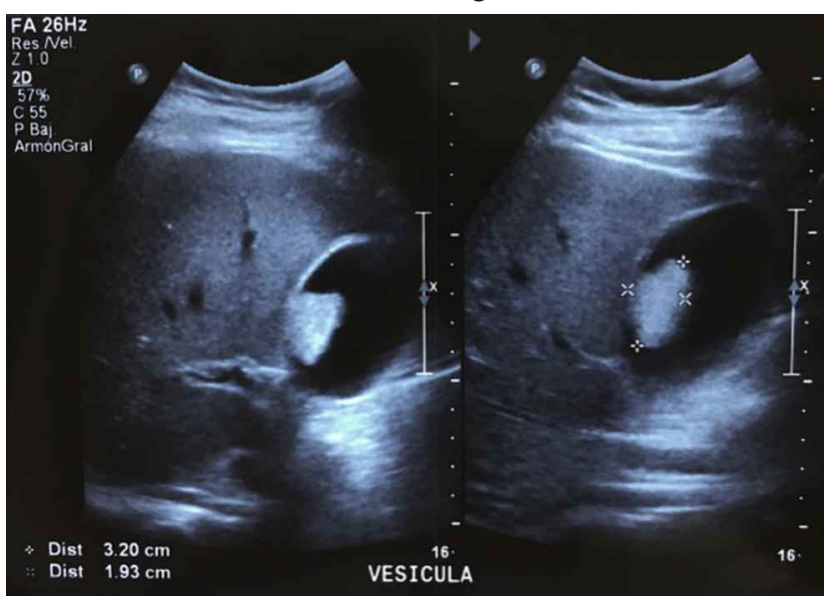

Fig. 1. Imagen ecotomográfica, en la que se puede observar una lesión poliposa de 32 x $19 \mathrm{~mm}$, localizada en el cuerpo de la vesícula biliar.

La exploración física y las pruebas de laboratorio habitual eran normales.
Dado el tamaño de la lesión, se programa para colecistectomía electiva, la que se realizó por vía laparoscópica el 11 de enero de 2018; con asistencia de anatomía patológica, para biopsia contemporánea de la lesión. La cirugía se pudo realizar sin inconvenientes, y al momento de la exploración laparoscópica, ya se logró evidenciar por trasparencia la presencia de la lesión (Fig. 2).

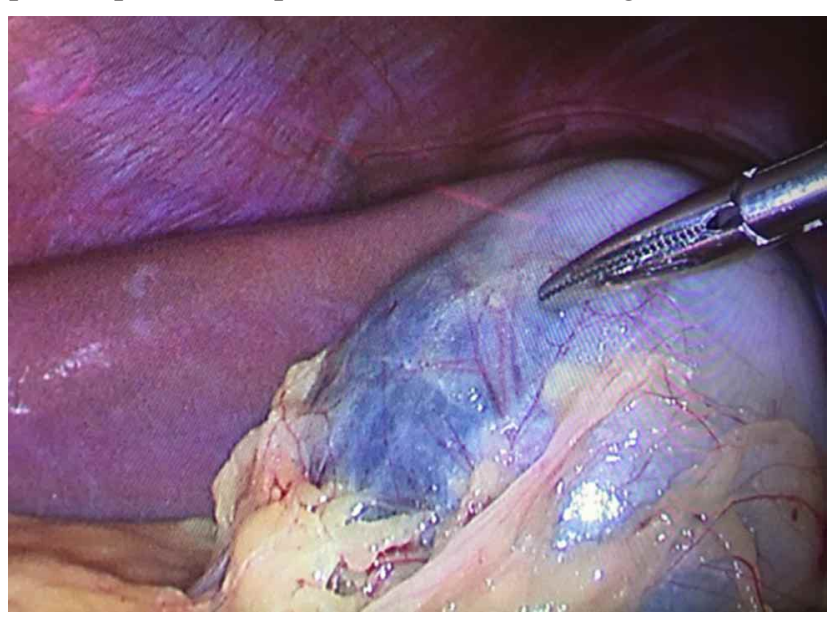

Fig. 2. Imagen laparoscópica, en la que se puede apreciar el margen libre de la vesícula biliar y por trasparencia, la lesión.

Una vez extirpado el espécimen, éste fue abierto y observado al fresco, momento en el que se verificó la apariencia y características del pólipo (Fig. 3A), que tenía una pequeña base de implantación (Fig. 3B), a partir de la cual se tomaron muestras para biopsia por congelación, que fue negativa para infiltración neoplásica (sugerente de neoplasia papilar intravesicular); razón por la que la cirugía se concluyó, por considerarse suficiente para la patología del paciente.

El paciente evolucionó de forma adecuada y fue dado de alta al día siguiente de la intervención, sin problemas.

El resultado definitivo del estudio histopatológico (incluido el ulterior mapeo de la vesícula biliar) (Fig. 4), fue de NPIC, colecistitis crónica inespecífica, colesterolosis y pólipos colesterínicos (Figs. 3A-B, 5); con base en la observación de pared de vesícula biliar de arquitectura distorsionada por lesión corporal exofítica constituida por proliferación de estructuras glandulares tubulares y papilares, con desorden citoarquitectural, núcleos hipercromáticos, elongados, con tendencia a la estratificación y en partes cromatina grumosa, con escaso citoplasma basófilo. Negativo para infiltración en la base de implantación de la lesión y de la pared vesicular por estructuras glanduliformes atípicas. En el corion de la mucosa se constató infiltrado inflamatorio linfoplasmocitario con acúmulos de histiocitos espumosos. Además, se reconocieron 5 estructuras polipoide revestidas por epitelio columnar normotípico, con acúmulos de histiocitos espumosos en el corion de la mucosa (Fig. 5). 
En la actualidad, el paciente lleva 8 meses de seguimiento clínico y con imágenes, sin evidencias de recidiva.
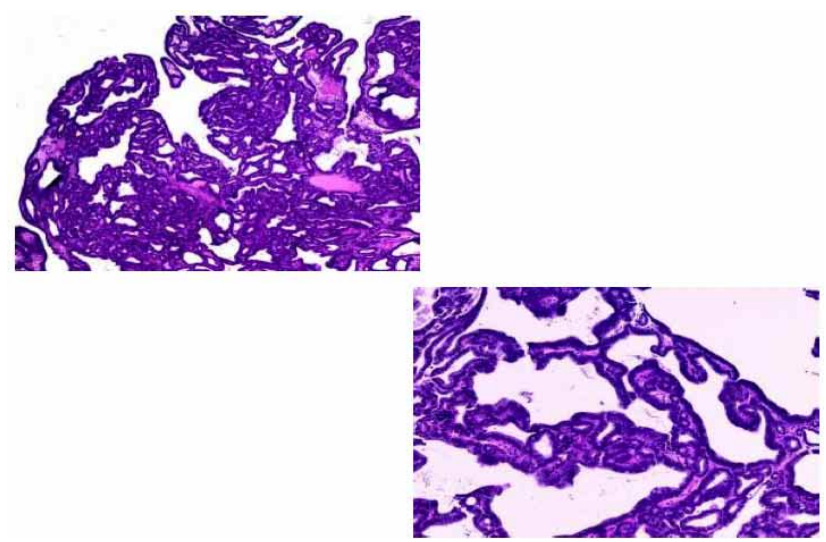

Fig. 3. Imágenes del espécimen vesicular abierto por su magen hepático $(9,9$ por $5,5 \mathrm{~cm})$. A) Se puede observar a nivel del cuerpo lesión polipoide de $5 \mathrm{~cm}$ de longitud y de entre 1 y $1,5 \mathrm{~cm}$ de diámetro, de superficie pardo-rosada, lisa y friable. Además, pequeños pólipos colesterínicos de 0,1 a a $0,3 \mathrm{~cm}$. B) Una vez extendida la lesión, se constata que esta pende de una pequeña base localizada en la unión del cuerpo con el fondo vesicular.

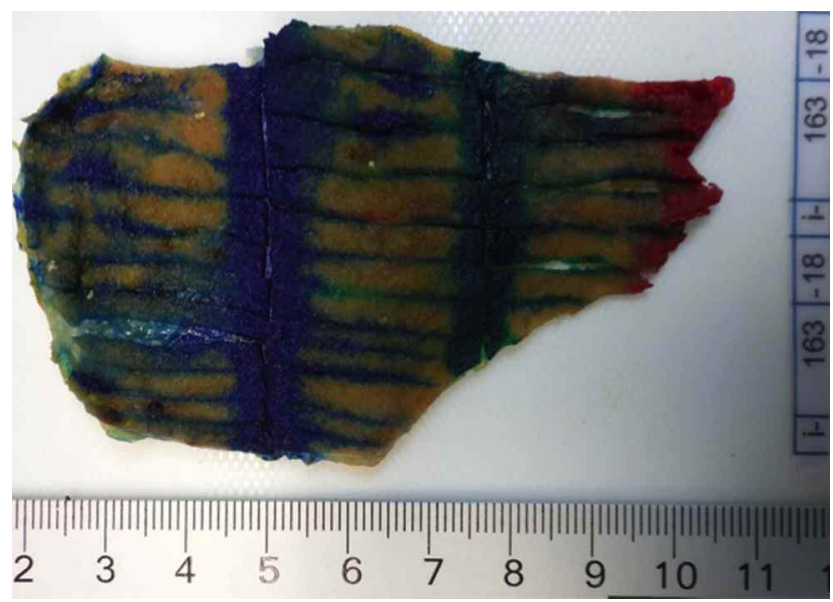

Fig. 4. Imagen del mapeo de la vesícula biliar del paciente.

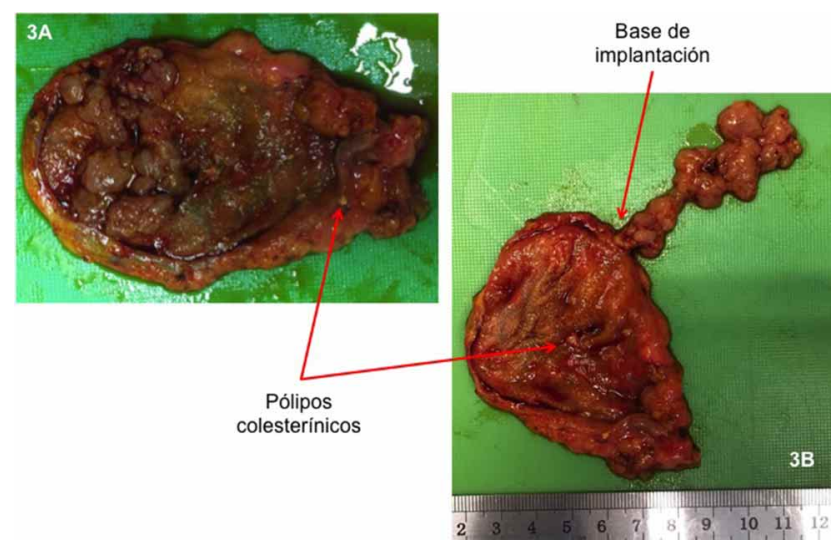

\section{DISCUSIÓN}

La NPIC es una entidad recientemente establecida, descrita por primera vez en 2010 en la clasificación de la Organización Mundial de la Salud (Adsay et al.). Previamente era conocida como adenoma/carcinoma papilar. Se trata de un tumor poco frecuente que forma una masa polipoide o papilar intramucosa a menudo asociada a diferentes cantidades de mucina. Posteriormente, fue descrita como una masa intramucosa exofítica en la vesícula biliar, mayor de $1 \mathrm{~cm}$, compuesta de células displásicas formando una lesión distinta a la mucosa circundante (Adsay et al.). Se considera como una lesión precursora para el desarrollo de carcinoma de vesícula biliar invasivo, al igual que la BilIN (Bennett et al.). Dicha neoplasia se considera como análoga al TPMI.

Se presenta con mayor frecuencia en mujeres, con una edad media de 60 años. Es frecuentemente asintomática, aunque, si la comparamos con BillN, suele estar más asociada a síntomas tempranos de dolor intermitente, ictericia o colangitis (Bennett et al.).

La NPIC se encuentra entre el 0,3\% y 0,6\% de vesículas biliares intervenidas por colelitiasis o colecistitis, siendo usualmente un hallazgo incidental (Klöppel et al., 2013; Argon $e t$ al.); y se ha descrito que ocasionalmente, se puede asociar al síndrome de Peutz-Jeghers o al síndrome de Gardner (Páez Cumpa et al.).

Se han descrito 4 subtipos histológicos, análogos a los subtipos del TPMI: pancreatobiliar (el más frecuente), intestinal, gástrico y oncocítico (el de peor pronóstico). La NPIC usualmente presenta tanto áreas papilares como tubulares, pero si se considera el patrón de crecimiento predominante, cerca del $50 \%$ corresponde al patrón papilar. Este tiende a tener un fenotipo biliar, mientras que el fenotipo gástrico está mayormente asociado a patrones tubulares. La displasia de alto grado es más común en el patrón papilar y túbulo-papilar. El subtipo pancreatobiliar típicamente muestra expresión CK7 y MUC1, mientras que el subtipo gástrico suele expresar MUC5AC y MUC6, el intestinal expresa MUC2, CDX2 y CK20, y el oncocítico, MUC1. La tinción inmunohistoquímica MUC1 se ha descrito como un marcador de mal pronóstico en el NPIC (Adsay et al.).

Fig. 5. Imagen de la histopatología de la lesión. Pared de vesícula biliar de arquitectura distorsionada por lesión corporal exofítica constituida por proliferación de estructuras glandulares tubulares y papilares, con desorden citoarquitectural, núcleos hipercromáticos, elongados, con tendencia a la estratificación y escaso citoplasma basófilo. 
El $6 \%$ de los carcinomas de vesícula biliar invasivos provienen de la NPIC. La tasa de carcinoma invasivo se encuentra en torno al $60 \%$. Los pacientes con NPIC no invasiva tienen una tasa de supervivencia a los 5 años de un $80 \%$, la cual se reduce al $60 \%$ en casos asociados a carcinoma invasivo. Se ha descrito en algunos estudios que el carcinoma invasivo asociado a NPIC tiene un mejor pronóstico que aquel asociado a BilIN (Klöppel et al.).

El tratamiento es la resección quirúrgica, requiriendo seguimiento posterior para detección temprana de lesiones asociadas, ya que, aunque no existen guías que protocolicen el seguimiento, se recomienda que los pacientes con NPIC sean seguidos de la misma manera que los que presentan el tipo clásico de cáncer de vesícula biliar, puesto que existe al menos un caso descrito en la literatura de desarrollo de metástasis hepáticas a los 16 meses del diagnóstico en un paciente con NPIC sin invasión vascular o linfática en el momento de la resección (Páez Cumpa et al.).

En resumen, presentamos un caso de NPIC en un paciente joven, cuyo diagnóstico fue confirmado por anatomía patológica tras una colecistectomía electiva, descartándose la presencia de carcinoma invasivo y displasia de alto grado, por lo que el pronóstico es favorable a pesar de presentar positividad para MUC1.

MANTEROLA, C.; BELLOLIO, E.; OTZEN, T. \& DUQUE, G. Intracholecystic papillary neoplasia. Report of a case and review of existing evidence. Int. J. Morphol., 36(4):1485-1489, 2018.

SUMMARY: Intracholecystic papillary neoplasm (ICPN) is a tumor composed of pre-invasive neoplastic cells, with up to $1.0 \mathrm{~cm}$ clinically detectable masses. The objective of this study was to report a case of NPIC and review the evidence in the literature. A 33-year-old asymptomatic male patient had an abdominal ultrasonography during a health examination, in which a vesicular polyp lesion of $32 \times 19 \mathrm{~mm}$ in diameter was verified. Thepatient was subsequently scheduled for elective cholecystectomy, which was performed laparoscopically and the surgery was uneventful. Once removed, the specimen was studied histopathologically and after performing vesicular mapping, we determined an ICPN, chronic non-specific cholecystitis, cholesteroliasis and cholesteric polyps. The patient has evolved without reported problems. We present a case of ICPN in a young patient, whose diagnosis was confirmed by pathological anatomy after an elective cholecystectomy, ruling out the presence of invasive carcinoma and high-grade dysplasia, with a favorable prognosis.

KEY WORDS: Intracholecystic Papillary-Tubular Neoplasms (ICPN); Neoplastic Polyps; Adenomas; Papillary Neoplasms; Gallbladder Neoplasm, Carcinoma in situ.

\section{REFERENCIAS BIBLIOGRÁFICAS}

Abraham, S. C.; Lee, J. H.; Hruban, R. H.; Argani, P.; Furth, E. E. \& Wu, T. T. Molecular and immunohistochemical analysis of intraductal papillary neoplasms of the biliary tract. Hum. Pathol., 34(9):902-10, 2003.

Adsay, V.; Jang, K. T.; Roa, J. C.; Dursun, N.; Ohike, N.; Bagci, P.; Basturk, O.; Bandyopadhyay, S.; Cheng, J. D.; Sarmiento, J. M.; Escalona, O. T.; Goodman, M.; Kong, S. Y. \& Terry, P. Intracholecystic papillary-tubular neoplasms (ICPN) of the gallbladder (neoplastic polyps, adenomas, and papillary neoplasms that are $\geq 1.0 \mathrm{~cm}$ ): clinicopathologic and immunohistochemical analysis of 123 cases. Am. J. Surg. Pathol., 36(9):1279-301, 2012.

Albores-Saavedra, J.; Tuck, M.; McLaren, B. K.; Carrick, K. S. \& Henson, D. E. Papillary carcinomas of the gallbladder: analysis of noninvasive and invasive types. Arch. Pathol. Lab. Med., 129(7):905-9, 2005.

Albores-Saavedra, J.; Vardaman, C. J. \& Vuitch, F. Non-neoplastic polypoid lesions and adenomas of the gallbladder. Pathol. Annu., 28 Pt. 1:145-77, 1993.

Aldridge, M. C. \& Bismuth, H. Gallbladder cancer: the polyp-cancer sequence. Br. J. Surg., 77(4):363-4, 1990

Almagro, U. A. Diffuse papillomatosis of the gallbladder. Am. J. Gastroenterol., 80(4):274-8, 1985.

Araki, T.; Hihara, T.; Karikomi, M.; Kachi, K. \& Uchiyama, G. Intraluminal papillary carcinoma of the gallbladder: prognostic value of computed tomography and sonography. Gastrointest. Radiol., 13:261-5, 1988

Argon, A.; Barbet, F. Y. \& Nart, D. The relationship between intracholecystic papillary-tubular neoplasms and invasive carcinoma of the gallbladder. Int. J. Surg. Pathol., 24(6):504-11, 2016.

Arunabh; Sharma, A.; Shukla, N. K.; Mathur, M. \& Kapur, M. M. Diffuse papillomatosis of gall bladder. J. Assoc. Physicians India, 36(3):2289, 1988.

Bazira, L.; Thery, Y.; Habibou, A.; Tijdani, A.; Boukary, B. A.; Rakotomala, R. \& Illo, A. Diffuse papillomatosis of the gallbladder: case report of a hydropic gallbladder. Hepatogastroenterology, 45(24):2044-7, 1998.

Bennett, S.; Marginean, E. C.; Paquin-Gobeil, M.; Wasserman, J.; Weaver, J.; Mimeault, R.; Balaa, F. K. \& Martel, G. Clinical and pathological features of intraductal papillary neoplasm of the biliary tract and gallbladder. HPB (Oxford), 17(9):811-8, 2015.

Bhatt, N. R.; Gillis, A.; Smoothey, C. O.; Awan, F. N. \& Ridgway, P. F. Evidence based management of polyps of the gall bladder: A systematic review of the risk factors of malignancy. Surgeon, 14(5):278-86, 2016.

Diaz-Plasencia, J.; Vilela Desposorio, C.; Rebaza-Iparraguirre, H.; Villacorta Acosta, R. \& Calipuy Abanto, W. Polypoid lesions of the gallbladder. Rev. Gastroenterol. Peru, 14(1):22-6, 1994.

Escalona, A.; León, F.; Bellolio, F.; Pimentel, F.; Guajardo, M.; Gennero, R.; Cruz, J. P.; Viviani, P. \& Ibáñez, L. Gallbladder polyps: correlation between ultrasonographic and histopathological findings. Rev. Med. Chil., 134(10):1237-42, 2006.

Hashimoto, S.; Horaguchi, J.; Fujita, N.; Noda, Y.; Kobayashi, G.; Ito, K.; Koshida, S.; Kanno, Y.; Ogawa, T. \& Masu, K. Intracholecystic papillary-tubular neoplasm of the gallbladder presenting with jaundice. Intern. Med., 53(20):2313-7, 2014.

Kai, K. Organ-specific concept and controversy for premalignant lesions and carcinogenesis of gallbladder cancer. Hepatobiliary Surg. Nutr., 5(1):85-7, 2016.

Klöppel, G.; Adsay, V.; Konukiewitz, B.; Kleeff, J.; Schlitter, A. M. \& Esposito, I. Precancerous lesions of the biliary tree. Best Pract. Res. Clin. Gastroenterol., 27(2):285-97, 2013.

Kosemehmetoglu, K.; Akpinar, E.; Sokmensuer, C. \& Hamaloglu, E. Papillary carcinoma with diffuse papillomatosis of gallbladder and cystic duct. Ann. Diagn. Pathol., 15(2):140-4, 2011. 
Kozuka, S.; Tsubone, N.; Yasui, A. \& Hachisuka, K. Relation of adenoma to carcinoma in the gallbladder. Cancer, 50(10):2226-34, 1982.

Kushima, R.; Remmele, W.; Stolte, M. \& Borchard, F. Pyloric gland type adenoma of the gallbladder with squamoid spindle cell metaplasia. Pathol. Res. Pract., 192(9):963-9, 1996.

Lee, S. H.; Lee, D. S.; You, I. Y.; Jeon, W. J.; Park, S. M.; Youn, S. J.; Choi, J. W. \& Sung, R. Histopathologic analysis of adenoma and adenoma-related lesions of the gallbladder. Korean J. Gastroenterol., 55(2):119-26, 2010.

Michalinos, A.; Alexandrou, P.; Papalambros, A.; Oikonomou, D.; Sakellariou, S.; Baliou, E.; Alexandrou, A.; Schizas, D. \& Felekouras, E. Intracholecystic papillary-tubular neoplasm in a patient with choledochal cyst: a link between choledochal cyst and gallbladder cancer? World J. Surg. Oncol., 14(1):202, 2016.

Milchgrub, S.; Campuzano, M.; Casillas, J. \& Albores-Saavedra, J. Intraductal carcinoma of the pancreas. Cancer, 69(3):651-6, 1992

Nagahama, M.; Muto, Y.; Yamada, M.; Shiraishi, M.; Kusano, T. \& Toda, T. Villous adenoma of the gallbladder: a case report. Hepatogastroenterology, 44(15):681-4, 1997.

Nagata, S.; Ajioka, Y.; Nishikura, K.; Watanabe, G.; Inoue, T.; Yamaguchi, K.; Watanabe, H.; Tanaka, M. \& Tsuneyoshi, M. Co-expression of gastric and biliary phenotype in pyloric-gland type adenoma of the gallbladder: immunohistochemical analysis of mucin profile and CD10. Oncol. Rep., 17(4):721-9, 2007.

Nakajo, S.; Yamamoto, M. \& Tahara, E. Morphometrical analysis of gallbladder adenoma and adenocarcinoma with reference to histogenesis and adenoma-carcinoma sequence. Virchows Arch. A Pathol. Anat. Histopathol., 417(1):49-56, 1990.

Páez Cumpa, C.; Erimeiku Barahona, A.; Payeras Capó, M. A.; Amengual Antich, I. \& Garrido Durán, C. Hemobilia due to intracholecystic papillary neoplasm. Rev. Esp. Enferm. Dig., 109(1):70-3, 2017.

Resende, V.; Roda, R. \& Pedrosa, M. S. Gallbladder papillary neoplasia associated with intrahepatic carcinoma and pancreaticobiliary malformation. Gastroenterology Res., 5(6):245-8, 2012.

Roa, I.; de Aretxabala, X.; Araya, J. C. \& Roa, J. C. Preneoplastic lesions in gallbladder cancer. J. Surg. Oncol., 93(8):615-23, 2006.

Sessa, F.; Solcia, E.; Capella, C.; Bonato, M.; Scarpa, A.; Zamboni, G.; Pellegata, N. S.; Ranzani, G. N.; Rickaert, F. \& Klöppel, G. Intraductal papillary-mucinous tumours represent a distinct group of pancreatic neoplasms: an investigation of tumour cell differentiation and K-ras, p53 and c-erbB-2 abnormalities in 26 patients. Virchows Arch., 425(4):357-67, 1994.

Yamamoto, M.; Nakajo, S. \& Tahara, E. Histological classification of epithelial polypoid lesions of the gallbladder. Acta Pathol. Jpn., 38(2):181-92, 1988

\author{
Dirección para correspondencia: \\ Dr. Carlos Manterola Delgado \\ Departamento de Cirugía y CEMyQ \\ Universidad de La Frontera \\ Manuel Montt 112, oficina 408 \\ Temuco \\ CHILE
}

Email: carlos.manterola@ufrontera.cl

Recibido : 04-06-2018

Aceptado: 28-09-2018 